\title{
Invariant Metrics, An Important Tool in Several Complex Variables
}

\author{
Marek Jarnicki ${ }^{1}$ - Steven G. Krantz ${ }^{2}$. Peter Pflug ${ }^{3}$
}

Published online: 7 January 2020

(c) Mathematica Josephina, Inc. 2020

Invariant distances and metrics like the Carathéodory distance, the CarathéodoryReiffen metric, the Kobayashi distance, the Kobayashi-Royden metric, the Azukawa metric, and the Bergman distance/metric played and play a prominent role in understanding properties of the geometry in Several Complex Variables. This special issue contains various contributions written by experts in that area. Some articles are surveys, other contain recent results. All of them present a broad view on how these objects are used effectively to get a deeper understanding of complex geometry. We would like to thank all the authors who spent their time and effort on creating a strong insight into this area of mathematics.

M.J. and P.P. would like to thank Professor Steven G. Krantz for his idea of having such a special issue and for giving us the opportunity to work together in this editorial project.

Publisher's Note Springer Nature remains neutral with regard to jurisdictional claims in published maps and institutional affiliations.

\footnotetext{
Marek Jarnicki

marek.jarnicki@im.uj.edu.pl

1 Jagiellonian University, Kraków, Poland

2 Washington University, St. Louis, USA

3 Carl von Ossietzky University, Oldenburg, Germany
} 